\section{Vascular scaffolding to seal giant aneurysms: a new technique}

Spontaneous rupture, fistula formation, thrombosis, distal embolisation and myocardial ischaemia are potential complications of coronary and vascular aneurysms. Several surgical techniques have been described to treat these aneurysms: ligation of the aneurysm, resection with end-to-end anastomosis, and vascular or coronary bypass grafting. Indications to treat coronary aneurysms remain unclear, and guidelines are not useful for making a decision. Treatment modalities have to be made by the clinician on a case-by-case basis. Renal and abdominal aneurysms' treatments are usually recommended for aneurysms over $15 \mathrm{~mm}$ of diameter. Stent-assisted coil embolisation has been reported for treatment of carotid and vertebrobasilar arteries. Recently, this treatment has been described for saccular and fusiform coronary aneurysm. We have tested this technique in patients with peripheral $(n=2)$ and coronary

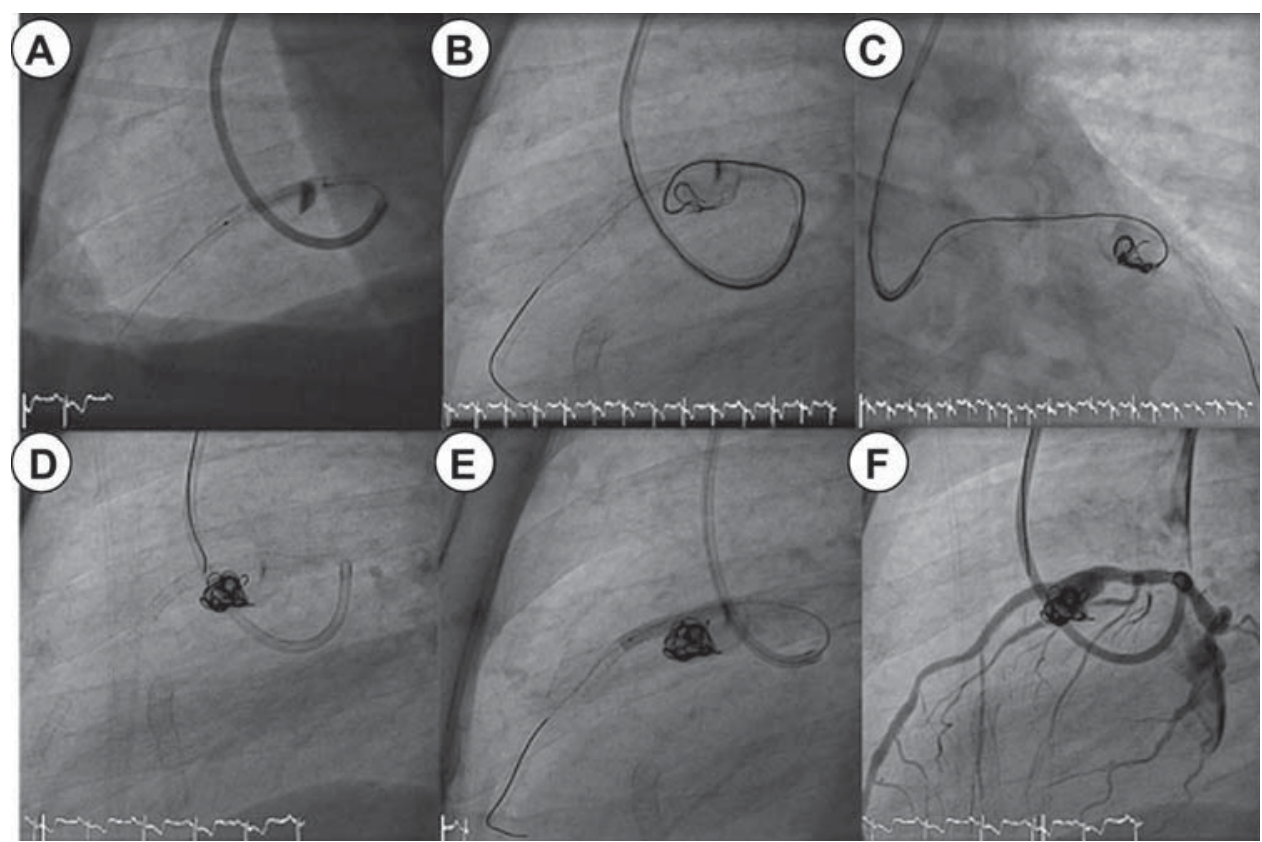

Figure 1 (A) Stent implantation at the site of the aneurysm. (B, C-F) Coil embolisation within the aneurysm, and final dilatation.

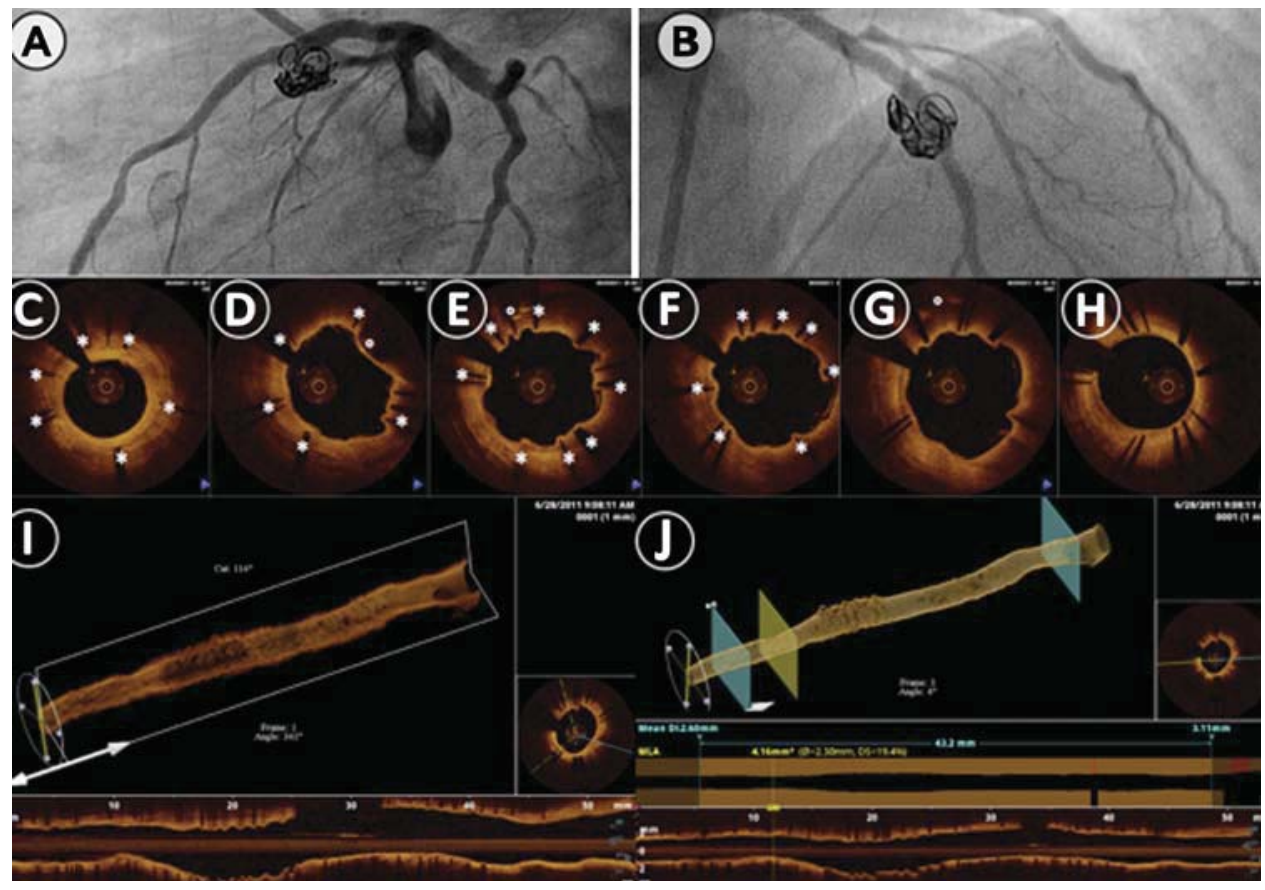

Figure 2 Six months angiographic $(A$ and $B)$ and OCT control $(C-J)$ showing a perfect healing of the aneurysm, with full exclusion and coverage of the stent's struts as shown by OCT imaging (view $\mathrm{C}-\mathrm{H}$ ). 
aneuryms $(\mathrm{n}=5)$ (figures 1 and 2 ) over the last 3 years. The technique consists in placing a bare metal stent (scaffolding effect) in the mother vessel using standard technique of stent deployment. A microcatheter is then inserted in the aneurysm through the struts of the stent, and coils are delivered via this microcatheter. To the best of our knowledge, this is the first report of a small series using this technique to treat vascular and coronary aneurysms. The procedure was uneventful in all patients, and at 6 months, the outcome was favourable with a full occlusion of the aneurysm and endothelialisation of the stent as confirmed by the OCT examination (figure 2).

\section{Jean Jacques Goy, Mario Togni, Stéphane Cook}

Service de Cardiologie, Hôpital Cantonal, Fribourg, Switzerland
Correspondence to Professor Jean Jacques Goy, Service de Cardiologie, University \& Hospital Fribourg, Rue des Pensionnats 3, 1700 Fribourg, Switzerland; ijgoy@goyman.com

Contributors All authors were involved in the procedure, participated in the elaboration of the manuscript, and approved it.

Funding This work was supported by Fondation de cardiologie Fribourg. Competing interests None.

Ethics approval Ethics approval was provided by Hospital Ethical Committee. 